\section{Auto-estima e fatores associados em gestantes da cidade de Pelotas, Rio Grande do Sul, Brasil}

\author{
Self-esteem and associated factors in pregnant \\ women in the city of Pelotas, Rio Grande do Sul \\ State, Brazil
}

\author{
${ }^{1}$ Faculdades Atlântico Sul/ \\ Anhanguera Educacional, \\ Pelotas, Brasil. \\ 2 Programa de Pós-graduação \\ em Saúde e Comportamento, \\ Universidade Católica de \\ Pelotas, Pelotas, Brasil. \\ 3 Curso de Psicologia, \\ Universidade Católica de \\ Pelotas, Pelotas, Brasil. \\ Correspondência \\ M. S. Dias \\ Faculdades Atlântico Sul/ \\ Anhanguera Educacional. \\ Rua Dr. Bruno Chaves 300 , \\ Bloco G, apto. 107, Pelotas, RS \\ 96055-040, Brasil. \\ michelle_souzadias@hotmail. \\ com
}

\author{
Michelle de Souza Dias ${ }^{1}$ \\ Ricardo Azevedo da Silva ${ }^{2}$ \\ Luciano Dias de Mattos Souza 3 \\ Rosângela da Costa Lima 2 \\ Ricardo Tavares Pinheiro ${ }^{2}$ \\ Inácia Gomes da Silva Moraes 3
}

\section{Introdução}

This study analyzes self-esteem and associated factors in pregnant women treated by the Unified National Health System (SUS) in the city of Pelotas, Rio Grande do Sul State, Brazil. Using a cross-sectional design, 560 pregnant women were interviewed from May to November 2006. The interviews were held in specific locations like University outpatient clinics and hospital wards and a center run by the city government. A full 62.9\% were diagnosed as high-risk pregnancies. Mean self-esteem according to the Rosenberg scale was $9.2(S D=4.6)$. Variables showing a positive, significant association with self-esteem were age, schooling, and income. Perception of risk to the unborn infant's health and parity were both negatively associated with maternal self-esteem. These high-risk pregnant women also showed higher self-esteem than low-risk pregnant women.

Self Concept; High-Risk Pregnancy; Pregnant Women
A gestação é um período de peculiar suscetibilidade para as mulheres, sendo com freqüência estressante física e mentalmente 1. Mesmo na gravidez saudável, alterações físicas e emocionais podem modificar a habilidade da mulher administrar suas funções e papéis usuais ${ }^{2}$. Além disso, é importante salientar que os sintomas psicopatológicos durante a gravidez têm conseqüências fisiológicas tanto para a mãe ${ }^{3}$ quanto para o bebê 4 .

A gestação é um fenômeno fisiológico e, por isso mesmo, sua evolução se dá na maior parte dos casos com baixo-risco. Apesar deste fato, há parcela de gestantes que, por características específicas, ou por algum agravo, apresenta maiores probabilidades de evolução desfavorável, tanto para o feto como para a mãe. Essa parcela constitui o grupo chamado de "gestante de altorisco" 5 .

Uma gravidez é considerada de alto risco quando apresenta alguma intercorrência de ordem materna ou fetal durante o período de desenvolvimento intra-uterino do concepto, afetando a evolução e o resultado da gravidez ${ }^{6}$. Um aspecto que pode prejudicar, em uma gestação de alto-risco, é a auto-estima das gestantes. A auto-estima é um conceito de grande abrangência, dizendo respeito à avaliação positiva ou negativa que o indivíduo faz de si mesmo, constituindose, assim, em um aspecto central do eu, que de- 
sempenha papel fundamental na construção da identidade adulta 7 . Além disso, é considerada geralmente o componente avaliativo do auto-conceito, uma representação mais larga do self, que inclui aspectos cognitivos e comportamentais, assim como avaliativos e afetivos 7. Ela é, portanto, uma conseqüência da identidade pessoal, congregando, dessa forma, as auto-avaliações sobre atributos e habilidades individuais manifestos nos domínios interpessoal e privado 8 .

Pouco se conhece a respeito dos aspectos psicológicos em mulheres com complicações perinatais, em especial sobre o efeito destas complicações na auto-estima. Um estudo de Kemp \& Page ${ }^{9}$ sobre este tema revelou diferenças significativas medidas pela escala de auto-estima de Rosenberg em mulheres experienciando uma gestação de alto-risco e uma gestação de baixorisco. Além disso, existem evidências de que a percepção de bem-estar durante a gravidez prediz positivamente o ajustamento do papel materno no período pós-natal 1,10,11.

No Brasil, são raros os estudos sobre as dificuldades psicológicas na gravidez de alto-risco e não existem estudos publicados sobre o reflexo na auto-estima das mulheres com alto-risco gestacional. Sendo assim, este estudo torna-se importante para a definição de estratégias de prevenção, ainda durante a gestação, de quadros mais graves com riscos para a saúde mental da mãe e, conseqüentemente, bem-estar do feto, uma vez que tem como objetivo analisar a autoestima e fatores associados em uma população de gestantes da cidade de Pelotas, Rio Grande do Sul, Brasil.

\section{Métodos}

O estudo se caracteriza por ser do tipo transversal, no qual foram estudadas 560 gestantes com diagnóstico de alto e baixo-risco, atendidas pelo sistema público de saúde nos três ambulatórios e duas enfermarias referência para esse tipo de patologia na cidade de Pelotas, durante os meses de maio a novembro de 2006.

Como este estudo faz parte de um estudo maior, em que o objetivo central era investigar a depressão em gestantes, utilizou-se o cálculo de tamanho de amostra para depressão.

Foram excluídas do estudo as mulheres que residiam em zona rural de Pelotas e/ou que manifestaram incapacidade de compreender ou responder o questionário.

As entrevistas foram feitas por 23 alunos do curso de Psicologia da Universidade Católica de Pelotas (UCPel), mediante processo de seleção e treinamento. A equipe contou também com a participação de seis alunos do curso de Medicina da mesma universidade, responsáveis pela confirmação dos diagnósticos de alto e baixo-risco das gestantes, treinados e supervisionados por dois médicos ginecologistas e obstetras.

As gestantes foram classificadas como sendo de alto ou baixo-risco por médicos do sistema público de saúde com base no manual técnico do Ministério da Saúde 5, que delimita quatro grupos de possíveis geradores de risco à gravidez, que são: características individuais e condições sócio-demográficas desfavoráveis, história reprodutiva anterior, doença obstétrica na gravidez atual e intercorrências clínicas. Neste estudo, foram desconsiderados os critérios sócioeconômicos e demográficos para diagnóstico de alto-risco. Essas variáveis foram, posteriormente, controladas na análise multivariada.

Para medir auto-estima, foi utilizada a escala de auto-estima de Rosenberg, validada para o Brasil por Dini 12. Este instrumento é composto de dez afirmativas com quatro opções de resposta que variam de concordo plenamente a discordo plenamente. As afirmativas de número um, três, quatro, sete e dez variando de concordo plenamente (valor $=0$ ) a discordo plenamente (valor $=3$ ) e as de número dois, cinco, seis, oito e nove variando de concordo plenamente (valor = 3 ) a discordo plenamente (valor $=0$ ). Dessa forma, o escore final da escala pode variar de 0 (melhor auto-estima) a 30 (pior auto-estima). Neste sentido, quanto maior a pontuação na escala, pior a auto-estima, e quanto menor a pontuação na escala, maior a auto-estima. No trabalho de avaliação da sensibilidade e comprovação da validade de construção dessa escala, apresentado por Dini 12, foram utilizados dois instrumentos, Self Reporting Questionnaire (SRQ-20) e Medical Outcomes Study 36-Item Short-Form Health Survey (SF-36), ambos mostraram-se significativos (valor de $\mathrm{p}<0,0001$ ) para a escala de auto-estima de Rosenberg pré e pós-cirurgia.

Para avaliar o bem-estar das gestantes, foi utilizada a escala de faces $13,14,15$, que é uma escala intervalar de sete pontos, com sete faces estilizadas; cada face consiste de um círculo com olhos que não mudam e uma boca com sorrisos que variam num contínuo de semi-círculo inclinado para cima até semi-círculo inclinado para baixo. Contudo, esta escala não teve a sua validade nem a sua reprodutibilidade testada no Brasil 14. As escalas visuais, no entanto, registram sentimentos inspirados pela experiência diária e garantem que todos os indivíduos percebem o mesmo estímulo visual 14, permitindo comparações intraindivíduos ao longo do tempo 16. A aplicação da escala de faces é simples e útil para estudos comunitários porque utiliza uma linguagem não- 
verbal - compreensível, portanto, em qualquer contexto cultural 14 .

Para levantar informações sobre o uso/abuso de álcool foi utilizada a escala CAGE. Desenvolvida por Mayfield et al. 17 e validada para o Brasil por Castells \& Furlanetto ${ }^{18}$, esta sinaliza suspeita de problemas com álcool (se pelo menos uma resposta das quatro perguntas for afirmativa - "sim") ou é indicativo deste (duas ou mais respostas afirmativas).

Para a classificação do nível econômico das participantes, foi utilizada a escala da Associação Brasileira de Empresas de Pesquisa (ABEP) 19.

No questionário foram inseridas outras variáveis identificadas na revisão de literatura: idade (em anos completos), estado marital (com ou sem companheiro), escolaridade (em anos completos), trabalho (sim ou não), religiosidade (se tinha alguma religião), para história obstétrica (número de gestações anteriores, se havia sido planejada, se foi desejada, se pensou em abortar, o número de consultas pré-natal), eventos estressores durante a gravidez (sim ou não), percepção de risco a sua saúde e à saúde do bebê (nenhum, pouco, moderado, alto risco) e internação hospitalar (no momento da aplicação do questionário). A história psiquiátrica individual foi investigada através de perguntas sobre e se havia feito tratamento psicológico ou medicamentoso para os nervos, o tabagismo (número de cigarros diários), uso de drogas (nos últimos trinta dias) e mal-estar psicológico (como se sentia a maior parte da gravidez) também foram investigados.

O estudo-piloto foi realizado nos meses de março e abril de 2006, com trinta gestantes, no qual os dados obtidos não foram incluídos na amostra final.

Foram realizadas visitas diárias aos três ambulatórios e nas duas enfermarias com o objetivo de identificar as gestantes que estivessem à espera de atendimento e posteriormente, para preenchimento da ficha de captação. Após o registro, os entrevistadores aplicavam os questionários nas gestantes que preenchiam os critérios de inclusão e que aceitaram participar do estudo, assinando previamente o consentimento livre e esclarecido. No caso das gestantes menores de idade, só foi realizada a aplicação do questionário com o seu consentimento livre e esclarecido e o de um responsável. Com relação às gestantes para as quais não foi possível aplicar o questionário ou com as quais não se terminou o processo de pesquisa no local do atendimento, a aplicação foi feita em um prazo máximo de trinta dias, na residência delas ou no retorno ao pré-natal.

Após as aplicações dos instrumentos, estes eram encaminhados à equipe responsável pela codificação e dupla digitação dos dados no programa Epi Info versão 6.04 (Centers for Disease Control and Prevention, Atlanta, Estados Unidos), com o objetivo de compará-las e detectar inconsistências dos dados. Como controle de qualidade, foi feito contato telefônico com $100 \%$ da amostra para se verificar a aplicação do questionário uma vez que este estudo faz parte de uma coorte que continuará analisando essas mulheres.

O processamento e análise dos dados foram realizados com o programa SPSS 10.0 (SPSS Inc., Chicago, Estados Unidos). Inicialmente, obtiveram-se as freqüências de todas as variáveis para caracterização da amostra. A seguir, foram calculadas as médias do escore de auto-estima em relação às variáveis independentes e comparadas, através do teste ANOVA, após o teste de homogeneidade de variância.

Posteriormente, foi realizada uma análise de regressão linear para identificar possíveis fatores de confusão. Foram avaliadas vinte variáveis no modelo de regressão inicial e as que estiveram associadas ( $\mathrm{p} \leq 0,20)$ com a exposição e o desfecho estudados foram incluídas no modelo hierárquico da análise multivariada. No primeiro nível do modelo foram incluídas as variáveis idade, escolaridade e nível econômico, no segundo, as variáveis percepção de risco à saúde da mãe e do bebê, número de gestações e internação hospitalar.

Este estudo foi aprovado pelos Comitês de Ética da UCPel e Universidade Federal de Pelotas (UFPel) e autorizado pela Secretaria de Saúde da Prefeitura Municipal de Pelotas.

\section{Resultados}

No total, 607 gestantes foram identificadas pela equipe, cinco se recusaram a participar do estudo e 17 foram consideradas perdas (não foram encontradas na residência após três visitas ou no retorno ao pré-natal em até um mês, ou o bebê nasceu ou morreu antes da aplicação do questionário), resultando num total de 585 gestantes.

Em relação à escala de auto-estima de Rosenberg, obteve-se o resultado de que das 585 gestantes - população estudada -, 560 responderam o instrumento utilizado e 25 foram consideradas perdas. Em relação ao diagnóstico de alto-risco, das 585 gestantes, três foram consideradas perdas, totalizando 582 das quais $37,1 \%$ foram consideradas baixo-risco e $62,9 \%$ alto-risco. Deste modo, as perdas e recusas somaram 47 gestantes, 7,7\% do total da população estudada.

A distribuição das variáveis incluídas no estudo pode ser observada na Tabela 1. A maioria das gestantes tinha entre 24 e 30 anos $(36,1 \%)$, era ca- 
Tabela 1

Distribuição das variáveis incluídas no estudo.

\begin{tabular}{|c|c|c|}
\hline Variáveis & Freqüência & Percentual válido \\
\hline \multicolumn{3}{|l|}{ Variáveis do primeiro nível } \\
\hline \multicolumn{3}{|l|}{ Idade (anos) } \\
\hline Até 18 & 99 & 17,7 \\
\hline $19-23$ & 140 & 25,0 \\
\hline $24-30$ & 202 & 36,1 \\
\hline 31 ou mais & 119 & 21,3 \\
\hline \multicolumn{3}{|l|}{ Estado marital } \\
\hline Não casada & 166 & 29,6 \\
\hline Casada ou vive com o companheiro & 394 & 70,4 \\
\hline \multicolumn{3}{|l|}{ Escolaridade } \\
\hline Até 1o grau completo & 275 & 49,4 \\
\hline 2o grau incompleto/completo & 250 & 44,9 \\
\hline Superior incompleto/completo & 32 & 5,7 \\
\hline \multicolumn{3}{|l|}{ Nível econômico } \\
\hline E e D & 206 & 37,9 \\
\hline C & 263 & 48,4 \\
\hline$B$ e A & 74 & 13,6 \\
\hline \multicolumn{3}{|l|}{ Trabalho } \\
\hline Não & 336 & 61,0 \\
\hline Sim & 215 & 39,0 \\
\hline \multicolumn{3}{|l|}{ Religião } \\
\hline Não tem & 144 & 25,8 \\
\hline Tem & 414 & 74,2 \\
\hline \multicolumn{3}{|l|}{ Variáveis do segundo nível } \\
\hline \multicolumn{3}{|l|}{ Idade gestacional (semanas) } \\
\hline Até 27 & 308 & 56,6 \\
\hline 28 ou mais & 236 & 43,4 \\
\hline \multicolumn{3}{|l|}{ Gestação atual } \\
\hline Planejada & 224 & 40,1 \\
\hline Aconteceu & 334 & 59,9 \\
\hline \multicolumn{3}{|l|}{ Pensou em abortar } \\
\hline Não & 512 & 91,9 \\
\hline Pensou & 45 & 8,1 \\
\hline \multicolumn{3}{|l|}{ Número de consultas pré-natal } \\
\hline Até 1 & 406 & 72,8 \\
\hline 2 & 86 & 15,4 \\
\hline 3 ou mais & 66 & 11,8 \\
\hline \multicolumn{3}{|l|}{ Percepção de risco à saúde da mãe } \\
\hline Nenhum risco & 356 & 63,9 \\
\hline Pouco ou moderado risco & 152 & 27,3 \\
\hline Alto risco & 49 & 8,8 \\
\hline \multicolumn{3}{|l|}{ Percepção de risco à saúde do bebê } \\
\hline Nenhum risco & 397 & 71,4 \\
\hline Pouco ou moderado risco & 122 & 21,9 \\
\hline Alto risco & 37 & 6,7 \\
\hline \multicolumn{3}{|l|}{ Número de gestações anteriores } \\
\hline Nenhuma & 247 & 44,1 \\
\hline 1 & 148 & 26,4 \\
\hline 2 & 75 & 13,4 \\
\hline 3 ou mais & 90 & 16,1 \\
\hline
\end{tabular}

(continua) 


\begin{tabular}{|c|c|c|}
\hline Variáveis & Freqüência & Percentual válido \\
\hline \multicolumn{3}{|l|}{ Internação hospitalar } \\
\hline Não & 459 & 84,5 \\
\hline Sim & 84 & 15,5 \\
\hline \multicolumn{3}{|l|}{ Evento estressor } \\
\hline Não & 226 & 40,6 \\
\hline Sim & 331 & 59,4 \\
\hline \multicolumn{3}{|c|}{ Tratamento psicológico ou medicamentoso para os nervos } \\
\hline Não & 401 & 73,0 \\
\hline Sim & 148 & 27,0 \\
\hline \multicolumn{3}{|l|}{ Escala CAGE } \\
\hline Abuso & 26 & 4,8 \\
\hline Não abuso & 515 & 95,2 \\
\hline \multicolumn{3}{|l|}{ Cigarros por dia } \\
\hline Nenhum & 482 & 86,1 \\
\hline Até 10 & 53 & 9,5 \\
\hline 11 ou mais & 25 & 4,5 \\
\hline \multicolumn{3}{|c|}{ Uso de drogas nos últimos trinta dias } \\
\hline Não usou & 529 & 97,2 \\
\hline Usou algum tipo de droga & 15 & 2,8 \\
\hline \multicolumn{3}{|l|}{ Mal-estar psicológico } \\
\hline Bem-estar & 510 & 92,6 \\
\hline Mal-estar & 41 & 7,4 \\
\hline \multicolumn{3}{|l|}{ Variável do terceiro nível } \\
\hline \multicolumn{3}{|l|}{ Condição gestacional } \\
\hline Baixo risco & 207 & 37,2 \\
\hline Alto risco & 350 & 62,8 \\
\hline
\end{tabular}

sada ou vivia com companheiro $(70,4 \%)$, possuía até o lo grau completo $(49,4 \%)$, pertencia à classe C (48,4\%), não trabalhava (61\%) e possuía alguma religião $(74,2 \%)$. De acordo com variáveis sobre a gestação atual, 56,6\% tinham até 27 semanas de gestação, 72,8\% fizeram pelo menos uma consulta pré-natal e $62,8 \%$ tinham o diagnóstico de alto-risco. Embora 59,9\% das gestantes tenham relatado que a gestação não foi planejada, a grande maioria, 91,9\%, não pensou em abortar e $59,4 \%$ relataram algum evento estressor. Em relação à percepção de risco, 63,9\% das gestantes não percebiam que a gestação trouxesse algum risco para a sua saúde e $71,4 \%$ não percebiam que a gestação trouxesse algum risco à saúde do bebê. Com relação à internação hospitalar, a grande parcela da população estudada não estava internada, $84,5 \%$. Cerca de $86 \%$ das gestantes referiram que não fumavam, não usaram drogas nos últimos trinta dias e não apresentaram abuso de álcool. Quase um terço destas mulheres faz ou já fez tratamento psicológico ou psiquiátrico, embora 92,6\% apresentassem bem-estar psicológico durante a gravidez. Os resultados referentes à avaliação da auto-estima, não incluídos na Ta- bela 1, mostraram uma média de 9,2, um desviopadrão de 4,6 e uma mediana de 9,0.

A Tabela 2 mostra que as gestantes mais jovens, com menor escolaridade, menor nível econômico, que não trabalhavam e não possuíam religião apresentavam a auto-estima significativamente menor. Também mostraram-se significativamente associados com menor auto-estima, os seguintes fatores: gestação não planejada, pensamento em abortar, perceber a gestação como trazendo alto risco para sua saúde e/ou do bebê, três ou mais gestações, algum evento estressor, tratamento psicológico ou medicamentoso para os nervos, não abuso de álcool, fumar 11 ou mais cigarros por dia, usar algum tipo de droga e mal-estar psicológico. As gestantes solteiras, com 28 semanas de gestação ou mais, que fizeram três ou mais consultas pré-natal, hospitalizadas e com diagnóstico de baixo-risco apresentaram pior auto-estima, embora a diferença não fosse significativa.

Os resultados da regressão linear são apresentados na Tabela 3. Outras variáveis investigadas como possíveis fatores de confusão que não estiveram associadas $(p>0,20)$ com a exposição 
Médias e desvio-padrão (DP) de auto-estima conforme variáveis independentes.

\begin{tabular}{|c|c|c|c|c|}
\hline Variável & $n$ * & Média & DP & Valor de $p$ ** \\
\hline Idade & & & & 0,00 \\
\hline Até 18 & 99 & 10,56 & 4,39 & \\
\hline $19-23$ & 140 & 9,74 & 4,26 & \\
\hline $24-30$ & 202 & 8,62 & 4,73 & \\
\hline 31 ou mais & 119 & 8,57 & 4,74 & \\
\hline Estado marital & & & & 0,10 \\
\hline Não casada & 166 & 9,73 & 4,73 & \\
\hline Casada ou vive com o companheiro & 394 & 9,02 & 4,55 & \\
\hline Escolaridade & & & & 0,00 \\
\hline Até 1o grau completo & 275 & 10,42 & 4,49 & \\
\hline 2o grau incompleto/completo & 250 & 8,30 & 4,31 & \\
\hline Superior incompleto/completo & 32 & 6,59 & 5,17 & \\
\hline Nível econômico & & & & 0,00 \\
\hline E e D & 206 & 10,59 & 4,65 & \\
\hline $\mathrm{C}$ & 263 & 8,52 & 4,36 & \\
\hline$B$ e $A$ & 74 & 7,57 & 4,21 & \\
\hline Trabalho & & & & 0,00 \\
\hline Não & 336 & 10,10 & 4,53 & \\
\hline Sim & 215 & 7,93 & 4,41 & \\
\hline Religião & & & & 0,00 \\
\hline Não tem & 144 & 10,48 & 4,94 & \\
\hline Tem & 414 & 8,79 & 4,41 & \\
\hline Idade gestacional (semanas) & & & & 0,73 \\
\hline Até 27 & 308 & 9,15 & 4,32 & \\
\hline 28 ou mais & 236 & 9,29 & 4,92 & \\
\hline Gestação atual & & & & 0,00 \\
\hline Planejada & 224 & 8,29 & 4,50 & \\
\hline Aconteceu & 334 & 9,85 & 4,58 & \\
\hline Pensou em abortar & & & & 0,00 \\
\hline Não & 512 & 9,03 & 4,55 & \\
\hline Pensou & 45 & 11,42 & 4,82 & \\
\hline Número de consultas pré-natal & & & & 0,78 \\
\hline Até 1 & 406 & 9,12 & 4,52 & \\
\hline 2 & 86 & 9,35 & 4,31 & \\
\hline 3 ou mais & 66 & 9,50 & 5,25 & \\
\hline Percepção de risco à saúde da mãe & & & & 0,01 \\
\hline Nenhum risco & 356 & 8,81 & 4,46 & \\
\hline Pouco ou moderado risco & 152 & 9,74 & 4,65 & \\
\hline Alto risco & 49 & 10,57 & 5,22 & \\
\hline Percepção de risco à saúde do bebê & & & & 0,00 \\
\hline Nenhum risco & 397 & 8,65 & 4,45 & \\
\hline Pouco ou moderado risco & 122 & 10,35 & 4,59 & \\
\hline Alto risco & 37 & 11,32 & 5,16 & \\
\hline Número de gestações anteriores & & & & 0,01 \\
\hline Nenhuma & 247 & 8,86 & 4,57 & \\
\hline 1 & 148 & 8,76 & 4,42 & \\
\hline 2 & 75 & 9,92 & 4,41 & \\
\hline 3 ou mais & 90 & 10,44 & 4,96 & \\
\hline Internação hospitalar & & & & 0,07 \\
\hline Não & 459 & 9,07 & 4,57 & \\
\hline Sim & 84 & 10,07 & 4,61 & \\
\hline
\end{tabular}

(continua) 


\begin{tabular}{|c|c|c|c|c|}
\hline Variável & $n$ * & Média & DP & Valor de $p$ ** \\
\hline Evento estressor & & & & 0,02 \\
\hline Não & 226 & 8,66 & 4,31 & \\
\hline Sim & 331 & 9,61 & 4,78 & \\
\hline \multicolumn{5}{|c|}{ Tratamento psicológico ou medicamentoso } \\
\hline para os nervos & & & 0,00 & \\
\hline Não & 401 & 8,76 & 4,41 & \\
\hline Sim & 148 & 10,59 & 4,89 & \\
\hline Escala CAGE & & & & 0,00 \\
\hline Abuso & 26 & 9,05 & 4,58 & \\
\hline Não abuso & 516 & 12,04 & 3,99 & \\
\hline Cigarros por dia & & & & 0,01 \\
\hline Nenhum & 482 & 9,01 & 4,56 & \\
\hline Até 10 & 53 & 10,26 & 4,78 & \\
\hline 11 ou mais & 25 & 11,28 & 4,55 & \\
\hline Uso de drogas nos últimos trinta dias & & & & 0,01 \\
\hline Não usou & 529 & 9,12 & 4,54 & \\
\hline Usou algum tipo de droga & 15 & 12,47 & 5,54 & \\
\hline Mal-estar psicológico & & & & 0,00 \\
\hline Bem-estar & 510 & 8,87 & 4,38 & \\
\hline Mal-estar & 41 & 13,90 & 4,67 & \\
\hline Condição gestacional & & & & 0,47 \\
\hline Baixo risco & 207 & 9,41 & 4,75 & \\
\hline Alto risco & 350 & 9,12 & 4,55 & \\
\hline
\end{tabular}

* Valores controlados para missings;

** Teste ANOVA.

e com o desfecho não foram incluídas na análise multivariada.

A análise ajustada mostrou que quanto maior a faixa etária, a escolaridade e o nível econômico, maior será a auto-estima. $\mathrm{O}$ decréscimo de pontos na escala de auto-estima foi, respectivamente, de 0,$45 ; 1,40$ e 0,97 , significando um crescimento da auto-estima.

As variáveis associadas negativamente à auto-estima foram percepção de risco à saúde do bebê e número de gestações anteriores. A cada mudança de categoria da percepção de risco à saúde do bebê, aumenta 1,11 pontos na escala, ou seja, piora a auto-estima da gestante. O maior o número de gestações anteriores também esteve significativamente associado com pior autoestima, verificou-se que a cada mudança de categoria (maior número de gestações) houve um acréscimo de 0,67 pontos.

As gestantes de alto-risco apresentaram significativamente melhor auto-estima do que aquelas de baixo-risco. A mudança de categoria de baixo-risco para alto-risco diminui 1,08 pontos na escala de auto-estima.

\section{Discussão}

As poucas investigações sobre o tema costumam enfatizar que os fatores psicossociais e biomédicos desempenham importante papel na percepção de risco de gestantes 20,21 e que o rótulo "alto-risco" pode afetar o estado psicossocial da mulher 22 .

Estudo de Moraes et al. 23, realizado com puérperas na mesma cidade verificou que as variáveis renda familiar e pensar em abortar estavam fortemente correlacionadas com a depressão pós-parto, ou seja, quanto pior a renda, maiores as chances de depressão pós-parto. No presente estudo o nível econômico também esteve associado à melhor auto-estima. Entretanto, o pensamento em abortar, que esteve associado na análise bruta, desapareceu na analise ajustada.

Assim como nesse estudo se encontrou uma maioria de mulheres casadas e que vivem com companheiro e com até o lo grau completo, Gupton et al. 20, em estudo com gestantes com e sem complicações e Hatmaker \& Kemp 24 em estudo com grávidas de baixo e alto-risco, também encontraram resultados similares, em 
Coeficientes de regressões conforme os subgrupos das variáveis independentes.

\begin{tabular}{|c|c|c|c|c|}
\hline Variáveis & Coeficiente bruto (IC95\%) & Valor de $p$ & Coeficiente ajustado (IC95\%) & Valor de $p$ \\
\hline \multicolumn{5}{|l|}{ Variáveis do primeiro nível } \\
\hline Idade (agrupada) & $-0,72(-1,10 ;-0,35)$ & 0,00 & $-0,45(-0,82 ;-0,70)$ & 0,02 \\
\hline Escolaridade (agrupada) & $-2,03(-2,65 ;-1,41)$ & 0,00 & $-1,40(-2,09 ;-0,71)$ & 0,00 \\
\hline Nível econômico (agrupado) & $-1,65(-2,21 ;-1,10)$ & 0,00 & $-0,97(-1,59 ;-0,35)$ & 0,00 \\
\hline \multicolumn{5}{|l|}{ Variáveis do segundo nível } \\
\hline Percepção de risco à saúde da mãe (agrupada) & $0,90(0,31 ; 1,45)$ & 0,00 & $-0,25(-0,99 ; 0,49)$ & 0,51 \\
\hline Percepção de risco à saúde do bebê (agrupada) & $1,48(0,85 ; 2,11)$ & 0,00 & $1,11(0,49 ; 1,72)$ & 0,00 \\
\hline Número de gestações (agrupada) & $0,53(0,19 ; 0,87)$ & 0,00 & $0,67(0,25 ; 1,08)$ & 0,00 \\
\hline Internação hospitalar & $1,00(-0,70 ; 2,07)$ & 0,07 & $-0,31(-1,37 ;-0,75)$ & 0,57 \\
\hline \multicolumn{5}{|l|}{ Variável do terceiro nível } \\
\hline Condição gestacional (alto-risco) & $-0,29(-1,09 ; 0,51)$ & 0,47 & $-1,08(-1,87 ;-0,30)$ & 0,01 \\
\hline
\end{tabular}

relação à situação conjugal e/ou marital e escolaridade das mulheres.

Thornburg 25 relata a experiência da gestação de alto-risco como uma prolongada vigília de trabalho sobrecarregado na percepção de grávidas hospitalizadas. Nesta direção alguns estudos mostram que os níveis de ansiedade são significativamente maiores em gestantes de alto-risco do que nas consideradas de baixo risco 21,26,27. Finalmente, Kemp \& Page 9 mostraram diferenças significativas na auto-estima medida pela escala de auto-estima de Rosenberg em mulheres experienciando uma gestação de alto-risco e em mulheres com baixo-risco, além de ter encontrado associação com o planejamento da gestação. Gupton et al. 20 em seu estudo também revelou que as gestantes com complicações apresentavam uma pior auto-estima, quando comparadas com as gestantes sem complicações. A presente investigação encontrou associação entre autoestima e diagnóstico de alto-risco na direção oposta, revelando que as gestantes com a condição gestacional de alto-risco apresentam menos pontos na escala indicando melhor auto-estima do que as de baixo-risco. Além disso, ao investigar o impacto da hospitalização na auto-estima, não se encontrou diferença significativa entre gestantes hospitalizadas e não hospitalizadas e também não se encontrou significância com o planejamento da gestação. Cabe salientar que os estudos acima mencionados possuíam amostras pequenas e de conveniência, não tendo a amplitude do presente estudo.

Segundo Souza \& Ferreira 28, a auto-estima pessoal e auto-estima coletiva de mulheres que possuem filhos são significativamente maiores do que os índices obtidos por mulheres que não possuem filhos. Mulheres não-mães sentem-se desvalorizadas 29 e apresentam índices de bemestar subjetivo 30 e de auto-estima inferiores 31 , quando comparadas às mulheres mães. Assim, o fato de ter um filho ou estar grávida pode contribuir positivamente para a auto-estima da gestante. O presente estudo, que trata só de gestantes, encontrou associação significativa com o número de gestações. Notou-se que na medida em que aumenta o número de gestações anteriores, aumenta a pontuação na escala, diminuindo a auto-estima.

O estudo de Gupton et al. 20 afirma que as grávidas com complicações percebem sua gestação como lhes trazendo risco. O presente estudo mostrou associação significativa entre a condição gestacional de baixo e alto-risco e a percepção, pela própria gestante, de a gravidez trazer algum risco para si e para o bebê quando analisadas individualmente com as variáveis auto-estima e condição gestacional, mas na análise ajustada, só permaneceu significativa a percepção de risco à saúde do bebê.

O estudo de Stevenson et al. ${ }^{32}$, com grávidas adolescentes, e o estudo de Kuehner \& Buerger 33 com pacientes deprimidos, encontraram correlação significativa entre auto-estima e bem-estar, já o presente estudo encontrou correlação entre auto-estima e bem-estar em análise bivariada com auto-estima e não com condição gestacional, não sendo incluída na análise ajustada.

A presença de auto-estima elevada tem sido relacionada a baixos níveis de tensão emocional e depressão 34,35 , inclusive Hall et al. 36 em estudo com 738 mulheres no período pós-parto, encontraram o dado que a mulheres com baixa 
auto-estima têm provavelmente 39 vezes mais chances de apresentarem sintomas depressivos quando comparadas com mulheres com alta auto-estima, sendo assim, um estudo acerca da auto-estima em gestantes se faz necessário para pensarmos em estratégias de prevenção de depressão pós-parto. Além disso, o conceito de auto-estima tem sido usado como sinônimo de auto-eficácia 37 , indicando, portanto o sentimento de confiança que o indivíduo tem de realizar tarefas propostas a ele, o que para gestantes é importante, à medida que devem sentir-se confiantes para assumir o papel materno.

A hipótese de que as gestantes de alto-risco apresentariam uma pior auto-estima, quando comparadas com as de baixo-risco, não foi confirmada, talvez, porque as gestantes de alto-risco recebam um maior número de atendimentos, com pré-natais quinzenais no início da gestação e semanais no final, diferente das gestantes de baixo-risco, que no início fazem pré-natal mensal e no final da gestação, quinzenal. Nesse sentido, deve-se enfatizar o efeito hawthorne - efeito produzido no paciente pelo aumento do interesse sobre ele que pode provocar mudança de comportamento. Dessa forma, podemos supor que quando a pessoa é mais cuidada, ela tende a melhorar. Além disso, podemos supor que elas recebem mais apoio e cuidados da família e amigos.

\section{Resumo}

O estudo tem como objetivo mensurar a auto-estima e fatores associados em gestantes atendidas pelo Sistema Único de Saúde (SUS) na cidade de Pelotas, Rio Grande do Sul, Brasil. É do tipo transversal, tendo-se entrevistado 560 gestantes nos meses de maio a novembro de 2006, nos ambulatórios e enfermarias dos hospitais universitários e do centro de atendimento da Prefeitura Municipal. Dentre as gestantes atendidas, 62,9\% receberam o diagnóstico de alto-risco. Para avaliação da auto-estima foi utilizada a escala de Rosenberg. Na amostra total a média de pontos na escala foi de 9,2 e o desvio-padrão de 4,6. As variáveis associadas positiva e significativamente com auto-estima foram idade, nível de escolaridade e nível econômico. Já as variáveis percepção de risco à saúde do bebê e número de gestações mostraram-se associadas negativamente à auto-estima. Além disso, as gestantes com condição gestacional de alto-risco têm uma auto-estima mais elevada quando comparadas com as de baixo-risco.
Contudo, cabe salientar que este não é um estudo populacional, excluindo da amostra eventuais gestantes de alto-risco não atendidas nos locais de referência a esse tipo de patologia e gestantes que recebem atendimento exclusivamente particular ou de convênio. Além disso, verificou-se que o diagnóstico da condição gestacional como sendo de alto-risco é complexo. O presente estudo, sendo transversal, não permite compreender o desenvolvimento do tema em relação ao longo do tempo. Ainda apresenta a limitação de desconhecer se há auto-estima das gestantes antes da gravidez, não permitindo avaliar se a mulher já possuía uma auto-estima baixa ou alta antes mesmo da gestação.

Dessa forma, pode-se concluir que esse estudo encontrou resultados inovadores sobre a auto-estima em gestantes, principalmente quando falamos em condição gestacional de alto-risco, ou seja, essas mulheres, ao contrário do que se pensava, não apresentam pior auto-estima do que as de baixo-risco, dados indicados em outros estudos, que apresentavam amostras pequenas $\mathrm{e}$ de conveniência, não tendo a amplitude do presente estudo. Sendo assim, a condição gestacional de alto-risco por si só não implica em baixa auto-estima nas mulheres, podendo se pensar que a ênfase que se dá às patologias seja muito maior do que o efeito destas na auto-estima das gestantes.

\section{Colaboradores}

M. S. Dias, R. A. Silva e I. G. S. Moraes coordenaram a investigação, conceberam o presente artigo e realizaram a revisão bibliográfica. R. C. Lima elaborou e revisou a análise estatística do artigo. L. D. M. Souza e R. T. Pinheiro supervisionaram o trabalho de campo e a ação dos entrevistadores, além da elaboração do texto final. R. A. Silva e R. T. Pinheiro realizaram a revisão final. 


\section{Referências}

1. Rofé Y, Blittner M, Lewin I. Emotional experiences during the three trimesters of pregnancy. J Clin Psychol 1993; 49:3-12.

2. Gjerdingewn D, Froberg DG, Fontaine P. The effects of social support on women's health during pregnancy, labor and delivery, and postpartum period. Fam Med 1991; 23:370-5.

3. Wadhwa PD, Dunkel-Schetter C, Chicz-DeMet A, Porto M, Sandman CA. Prenatal psychosocial factors and the neuroendocrine axis in human pregnancy. Psychosom Med 1996; 58:432-46.

4. Teixeira JMA, Fisk NM, Glover V. Association between maternal anxiety in pregnancy and increased uterine artery resistance index: cohort based study. BMJ 1999; 318:153-7.

5. Ministério da Saúde. Gestação de alto risco: manual técnico. 3a Ed. Brasília: Ministério da Saúde; 2000.

6. Montenegro CAB, Rezende J. Obstetrícia fundamental. 5a Ed. Rio de Janeiro: Editora Guanabara Koogan; 1987.

7. Blascovich J, Tomaka J. Measures of self-esteem. In: Robinson JP, Shaver P, Wrightsman LS, editors. Measures of personality and social psychological attitudes. v. I. San Diego: Academic Press; 1991. p. 115-55.

8. Páez D, Martinez-Taboada C, Arróspide JJ, Insúia P, Ayestarán S. Constructing social identy: the role of status, collective values, collective self-esteem, perception and social behavior. In: Worchel S, Morales JF, Páez D, Deschamps J-C, editors. Social identy: international perspectives. London: Sage Publications; 1998. p. 211-29.

9. Kemp VH, Page C. Maternal self-esteem and prenatal attachment in high-risk pregnancy. Matern Child Nurs J 1987; 16:195-206.

10. Paarlberg KM, Vingerhoets AJ, Passchier J, Decker GA, Heinen AG, van Geijin HP. Psychosocial factors and pregnancy outcome: a review with emphasis on methodological issues. J Psychosom Res 1995; 39:563-95.

11. Mothander PR. Maternal adjustment during pregnancy and the infant's first year. Scand J Psychol 1992; 33:20-8.

12. Dini GM. Validade de construção e sensibilidade da escala de auto-estima de Rosenberg [Tese de Doutorado]. São Paulo: Escola Paulista de Medicina, Universidade Federal de São Paulo; 2004.

13. Andrews FM, Withey SB. Social indicators of wellbeing: Americans' perceptions of life quality. New York: Plenum; 1976.

14. McDowell I, Newell C. Measuring health: a guide to rating scales and questionnaires. Oxford: Oxford University Press; 1996.

15. Sparrenberger F, Santos I, Lima RC. Epidemiologia do distress psicológico: estudo transversal de base populacional. Rev Saúde Pública 2003; 37:434-9.

16. Altman DG. Practical statistics for medical research. 1st Ed. London: Chapman \& Hill; 1991.

17. Mayfield D, McLeod G, Hall P. The CAGE questionnaire: validation of a new alcoholism instrument. Am J Psychiatry 1974; 131:1121-3.
18. Castells MA, Furlanetto LM. Validade do questionário CAGE para rastrear pacientes com dependência ao álcool internados em enfermarias cínicas. Rev Bras Psiquiatr 2005; 27:54-7.

19. Associação Brasileira de Empresas de Pesquisa. Critério de classificação econômica do Brasil. São Paulo: Associação Brasileira de Empresas de Pesquisa; 2002.

20. Gupton A, Heaman M, Cheung LW. Complicated and uncomplicated pregnancies: women's perception of risk. J Obstet Gynecol Neonatal Nurs 2001; 30:192-201.

21. Hickey CA, Cliver SP, Goldenberg RL, McNeal SF, Hoffman HJ. Relationship of psychosocial status to low prenatal weight gain among nonobese black and white women delivering at term. Obstet Gynecol 1995; 86:177-83.

22. Stahl K, Hundley V. Risk and risk assessment in pregnancy: do we scare because we care? Midwifery 2003; 19:298-309.

23. Moraes IGS, Pinheiro RT, Silva RA, Horta BL, Sousa PLR, Faria AD. Prevalência da depressão pós-parto e fatores associados. Rev Saúde Pública 2006; 40:65-70.

24. Hatmaker D, Kemp V. Perception of threat and subjective well-being in low-risk and high-risk pregnant women. J Perinat Neonatal Nurs 1998; 12:1-10.

25. Thornburg P. "Waiting” as experienced by women hospitalized during the antepartum period. MCN Am J Matern Child Nurs 2002; 27:245-8.

26. Kmita G, Baranska M, Ciaston-Przeclawska E, Ceranska-Goszczynska H, Urmanska W. Psychological aspects of high risk pregnancy in hospitalized women: toward the change of the existing model of care. Ginekol Pol 1999; 69:1025-31.

27. Shennan A, Jones G, Hawken J, Crawshaw S, Judah J, Senior V, et al. Fetal fibronectin test predicts delivery before 30 weeks of gestation in high risk women, but increases anxiety. BJOG 2005; 112:293-8.

28. Souza D, Ferreira MC. Auto-estima pessoal e coletiva em mães e não-mães. Psicol Estud 2005; 10:19-25.

29. Remennick L. Childless in the land of imperative motherhood: stigma and coping among infertile Israeli women. Sex Roles 2000; 43:821-41.

30. Callan VJ, Hennessey JF. The psychological adjustment of women experiencing infertility. Br J Med Psychol 1998; 61:137-40.

31. Bailey N, Bruno MA, Bezerra LB, Queiroz T, Oliveira FN, Chen-Mok K. Adolescent pregnancy 1 years later: the effects of abortion vs. motherhood in northeast Brazil. J Adolesc Health 2001; 29:223-32.

32. Stevenson W, Maton K, Teti D. Social support, relationship quality, and well-being among pregnant adolescents. J Adolesc 1999; 22:109-21.

33. Kuehner C, Buerger C. Determinants of subjective quality of life in depressed patients: the role selfesteem, response styles, and social support. J Affect Disord 2005; 86:205-13. 
34. Logsdon MC, Usui, WM, Cronin SN, Miracle VA. Social support and adjustment in women following coronary artery bypass surgery. Health Care Women Int 1998; 19:61-70.

35. Vargas TP, Dantas RA, Góis CL. A auto-estima de indivíduos que foram submetidos à cirurgia de revascularização do miocárdio. Rev Esc Enferm USP 2005; 39:20-7.
36. Hall LA, Kotch JB, Browne D, Rayens MK. Self-esteem as a mediator of the effects of stressors and social resources on depressive symptoms in postpartum mothers. Nurs Res 1996; 45:231-8.

37. Stanley KD, Murpy MR. A comparison of general self-efficacy with self-esteem. Genet Soc Gen Psychol Monogr 1997; 123:81-99.

Recebido em 12/Nov/2007

Versão final reapresentada em 19/Mai/2008

Aprovado em 26/Mai/2008 